Journal of

Synchrotron

Radiation

ISSN 0909-0495

Received 9 July 2011

Accepted 30 September 2011

C 2012 International Union of Crystallography Printed in Singapore - all rights reserved

\section{A flow-through reaction cell for in situ X-ray diffraction and absorption studies of heterogeneous powder-liquid reactions and phase transformations}

\author{
Pilar Ferrer, ${ }^{\mathrm{a}, \mathrm{b} *}$ Iván da Silva, ${ }^{\mathrm{a}, \mathrm{b}}$ Juan Rubio-Zuazo, ${ }^{\mathrm{a}, \mathrm{b}}$ Belén F. Alfonso, ${ }^{\mathrm{c}}$ \\ Camino Trobajo, ${ }^{\mathrm{c}}$ Sergei Khainakov, ${ }^{\mathrm{c}}$ Jose R. Garcia, ${ }^{\mathrm{c}}$ Santiago Garcia-Granda ${ }^{\mathrm{c}}$ and \\ Germán R. Castro ${ }^{\mathrm{a}, \mathrm{b}}$
}

\begin{abstract}
aspLine Spanish CRG Beamline at the ESRF, European Synchrotron Radiation Facility, BP 220, 38043 Grenoble, France, ${ }^{\mathbf{b}}$ Instituto de Ciencia de Materiales de Madrid-ICMM/CSIC, Cantoblanco, Madrid 28049, Spain, and ${ }^{\mathbf{C}}$ Departamentos de Física, Química Física y Analítica y Química Orgánica e Inorgánica, Universidad de Oviedo, Oviedo, Spain. E-mail: ferreres@esrf.fr
\end{abstract}

A portable powder-liquid high-corrosion-resistant reaction cell has been designed to follow in situ reactions by X-ray powder diffraction (XRD) and X-ray absorption spectroscopy (XAS) techniques. The cell has been conceived to be mounted on the experimental stations for diffraction and absorption of the Spanish CRG SpLine-BM25 beamline at the European Synchrotron Radiation Facility. Powder reactants and/or products are kept at a fixed position in a vertical geometry in the X-ray pathway by a porous membrane, under forced liquid reflux circulation. Owing to the short pathway of the X-ray beam through the cell, XRD and XAS measurements can be carried out in transmission configuration/mode. In the case of the diffraction technique, data can be collected with either a point detector or a two-dimensional CCD detector, depending on specific experimental requirements in terms of space or time resolution. Crystallization processes, heterogeneous catalytic processes and several varieties of experiments can be followed by these techniques with this cell. Two experiments were carried out to demonstrate the cell feasibility: the phase transformations of layered titanium phosphates in boiling aqueous solutions of phosphoric acid, and the reaction of copper carbonate and Lisoleucine amino acid powders in boiling aqueous solution. In this last case the shrinking of the solid reactants and the formation of $\mathrm{Cu}$ (isoleucine) $)_{2}$ is observed. The crystallization processes and several phase transitions have been observed during the experiments, as well as an unexpected reaction pathway.

Keywords: powder-liquid reaction cell; cell design; in situ reaction; X-ray absorption spectroscopy; X-ray diffraction; layered titanium phosphates.

\section{Introduction}

Many important high-technology production processes take place on heterogeneous reactions systems. A very important aspect in order to improve many real scale industrial processes is a detailed understanding of the kinetics of the underlying heterogeneous reactions, including the morphological changes. The development of novel reaction process mechanisms is currently a very challenging process, as there is a significant lack of detailed understanding. Therefore, the main goals of today's research should be the tailored design of reaction processes and their end products, based on a fundamental understanding of the underlying reactions paths and intermediate products during heterogeneous reactions. To obtain these goals, an important aspect is the use of adequate techniques and experimental set-ups capable of producing detailed information during the reaction process. X-ray powder diffraction (XRD) and X-ray absorption spectroscopy (XAS) are two techniques that are very well placed to fulfil these requirements. X-ray powder diffraction is a rapid analytical technique, primarily used for phase identification of crystalline compounds by their diffraction pattern, and can provide information on unit-cell dimensions and average bulk composition. X-ray absorption spectroscopy is a useful technique particularly suitable for the study of the structural features of nanosystems, such as powder materials, amorphous and diluted systems. The strength of this spectroscopic technique is the capability to obtain the local electronic and geometrical structure of a system, without the necessity of presenting a long-range order. X-ray powder diffraction and 
X-ray absorption spectroscopy are also powerful tools for time-resolved in situ studies of phase transitions and reactions, since the electronic and crystallographic structure, including its morphology, can be obtained. However, even for these two suitable techniques, a special challenge is the case of powderliquid reactions where, normally, the reaction bath is permanently in circulation and therefore the solid parts, either for reactants or products, are in permanent movement. In this contribution a novel reaction cell for in situ study of temperature-controlled powder-liquid heterogeneous reactions is presented, which has the main advantage that the solid part is kept in the X-ray path in a fixed position. The cell has been designed to be mounted at the X-ray absorption spectroscopy stations and the six-circle multipurpose diffractometer of SpLine, the Spanish CRG BM25 beamline at the ESRF. In the latter case the set-up takes advantage of the twodimensional CCD detector mounted on the beamline. A typical full powder diffractogram can be recorded in a minimal time of $200 \mathrm{~ms}$, which defines the minimal kinetic timescale.

Many reactions require the presence of solvents to make possible or catalyze the solid phase transformation. Several varieties of cells have been developed to solve the needs that the heterogeneous reaction measurements require (Villain et al., 1993; Norby \& Hanson, 1998; Alison et al., 2003; Grunwaldt et al., 2005; FitzGerald et al., 2007; Wall et al., 2011). The main innovations for the cell presented in this work are: the fact that it can handle solid-liquid heterogeneous reactions keeping the powder reactants and/or products in the beam pathway in a fixed position during the chemical reaction, forced liquid solvent circulation through the powder assuring total powder-liquid contact, accurate temperature control in the range between 293 and $493 \mathrm{~K}$, and perfect compatibly with XRD and XAS experiments.

In order to show the cell capability, two heterogeneous solid-liquid reactions examples are presented. On one hand, the heterogeneous reaction of layered titanium phosphates in boiling aqueous solutions of phosphoric acid is studied by the $\mathrm{X}$-ray powder diffraction technique. Different molar concentrations of phosphoric acid allow the transformations between the $\alpha, \beta, \gamma$ and amorphous titanium phosphates phases. On the other hand, the $\mathrm{Cu}$-isoleucine complex synthesis has been followed by X-ray powder diffraction and X-ray absorption spectroscopy. The phase transformation of copper carbonate and L-isoleucine amino acid in boiling aqueous solution has been studied in situ and the complete reaction pathway of the $\mathrm{Cu}$ (isoleucine $)_{2}$ complex has been obtained.

\section{Beamline set-up}

$\mathrm{X}$-ray measurements (diffraction and absorption) have been carried out at the Spanish CRG SpLine-BM25 beamline at the European Synchrotron Radiation Facility (ESRF), in Grenoble, France. The beamline can tune the photon energy in the range 5-35 keV (wavelength 2.48-0.35 $\AA$ ) with an energy resolution of $\Delta E / E=10^{-4}$. Owing to the high flux $\left(\sim 10^{11}\right.$ photons s $\left.^{-1}\right)$ of the beamline and the two-dimensional $\mathrm{CCD}$ detector, time-resolved experiments are possible. High- resolution experiments are performed with a point detector which is mounted on the diffractometer arm. A $\theta-2 \theta$ set-up, together with a goniometer head, is installed in the detector arm in order to use a crystal analyzer for polarization analysis. The two-dimensional CCD detector is placed on a decoupled motorized stage. The CCD detector (Photonic Science CCD) is used to reduce the data acquisition time. The two-dimensional detector allows a short acquisition time for a whole powder diffraction pattern, showing the lack/presence of preferred orientation and/or large grain size. The twodimensional detector is a very high resolution large-area X-ray CCD array system that incorporates three tapered X-ray CCD modules. The camera produces a $250 \mathrm{~mm} \times 125 \mathrm{~mm} 33$ Mpixel resolution image with $32.8 \mu \mathrm{m}$ pixel size and a final resolution of $7651 \times 3825$ pixels. The three sensors are multiplexed in order to deliver a seamlessly stitched image at the user interface in $1.1 \mathrm{~s}$ with the option of further processing such as auto-flatfield and offset correction, exploiting the CCD interline structure (electronic shutter) of the Kodak KAI $11000 \mathrm{CCD}$. The accumulated charges are transferred to a non-sensitive area in microseconds, allowing new exposure while charges are transferred to the readout register. Simultaneous integration/readout enables $100 \%$ duty-cycle acquisition and increases the acquisition rate. The acquisition time can be varied as a function of binning and post-proceeding between a few milliseconds and $30 \mathrm{~min}$. In order to calibrate the wavelength and the sample-to-detector distance, a silicon standard powder is used, which is placed at the sample/cell position on the diffractometer. The powdered-sampledetector distance can be varied from a few centimetres up to $1500 \mathrm{~mm}$. This distance defines the area detector resolution. The CCD area detector can be rotated and placed with the larger sides either vertically or horizontally in order to maximize the accepted solid angle along or perpendicular to the synchrotron polarization plane. The use of a beam stopper is required to avoid detector damage occasioned by the direct $\mathrm{X}$-ray beam hitting the CCD detector.

The cell has been conceived to be mounted on the SpLineBM25 six-circle multipurpose diffractometer at the ESRF (Castro, 1998), taking advantage of the exceptional experimental set-up. The station, which is situated at branch B of the beamline, allows a great variety of techniques and experiments to be performed, with several available sample environments, as is the case of the powder-liquid reaction cell. The designed reaction cell has also been conceived to be mounted on the XAS station (Huber tower) located at branch $\mathrm{A}$ of the beamline, permitting $\mathrm{X}$-ray absorption experiments to be carried out.

\section{Solid-liquid reaction cell description}

The strength of the system is the tight integration of every component in the final design. The powder-liquid cell has been engineered to provide stable and precise performance on XAS and XRD measurements. The main body of the cell is built of Teflon material, which provides a high corrosion resistance, allowing a huge number of chemical reactions 
owing to the great variety of solvents that could be used. Moreover, the main body can be built on different materials, avoiding the limitations of the Teflon. The outside dimensions of the body are $125 \mathrm{~mm}$ length $\times 30 \mathrm{~mm}$ width and $51 \mathrm{~mm}$ height. The cell body is covered with stainless steel to offer physical resistance and it is used also as a metal heat protection. The cell dimension at the powder block support (X-ray pathway) is reduced to $4.5 \mathrm{~mm}$.

The novel cell has been designed taking into account that: (i) the powder must be kept in the X-ray impact position during the whole reaction process, and (ii) it has to be a short X-ray pathway, as the measurements are made in transmission mode. The powdered solid reactant is mounted in a special set-up which keeps the solid powder in the X-ray path during the chemical reaction. The liquid cavity has been designed to force the liquid circulation through the powder, as the vapour pressure in the cell increases, guaranteeing an optimum liquid-solid contact at the wished working conditions. The liquid circulates across the cell channel under reflux conditions. The X-ray pathway through the cell is minimized in order to reduce the X-ray absorption by the reaction bath formed by the liquid solvents and the solid powder (reactants and/or products). Two Kapton/Teflon windows of $5 \mathrm{~mm}$ aperture diameter are foreseen for the incoming and outcoming X-ray beam. The outcoming solid-angle aperture has been chosen to maximize the diffracted angular acceptation, covering a maximal angular range of $\pm 45^{\circ}$. The chosen angular acceptance allows a maximal $d$-space resolution of $0.3 \AA$ for a wavelength of $0.6 \AA$ (at the beamline photon critical energy of $20 \mathrm{keV}$ ). The $d$-space resolution can be adapted to the experimental requirements, changing either the detector-cell distance or the photon beam wavelength. The liquid temperature (and, consequently, the reaction temperature) is controlled between room temperature and $493 \mathrm{~K}$ by four cartridge heaters and a temperature thermocouple located in the cell body. The reaction temperature is limited by the material of the main body, i.e. for this reaction, Teflon. The cartridge heaters and the thermocouple are not in contact with the reaction bath. The liquid temperature is measured using a thermometer immersed in the reaction vessel. The cell is formed of three main parts (see Figs. 1 and 2): the powder holder, the body which contains the liquid channel (with the corresponding openings for distillation column, thermometer and the powder holder), and the protection/furnace cover. The powder holder is a Teflon block, which has a reception opening where the powder is positioned, covered by a porous membrane envelope. The powder holder block is located in the middle of the Teflon body. Hence, the powder is kept in the beam position by the porous membrane, whose porous size is lower than the grain size of the powder but high enough to assure the liquid circulation. The powder holder has additional openings to facilitate the liquid flow and, depending on the membrane's porous dimensions (available, for example, from Goodfellow), powder from $500 \mathrm{~nm}$ to $50 \mu \mathrm{m}$ grain size can be studied with an optimum contact with the flow solution.

In Fig. 1 a scheme of the cell is shown. In the interior cavity there is a channel where the liquid flows along the main body. The solvent channel is connected to the exterior through seven exits: three in the upper, two in the central and two in the lateral positions. At the top there are three main holes: two in conical form (14/23), which are foreseen to locate a control thermometer or a liquid reservoir (left) and a distillation column (right) to guarantee the reflux conditions, and one elongated central aperture of $21 \mathrm{~mm} \times 3 \mathrm{~mm}$ (length $\times$ width) and $32 \mathrm{~mm}$ depth which is used to position the powder holder. The incorporation of the liquid solvents is made through one of the conical apertures of the top side. In the middle of the long side of the cell body, a $12 \mathrm{~mm}$ cylindrical aperture is used for the incident X-ray beam. At each of the cell long sides an additional $20 \mathrm{~mm}$ outlet disc is placed to allocate a metal window support. On the short sides, two extra holes are made 


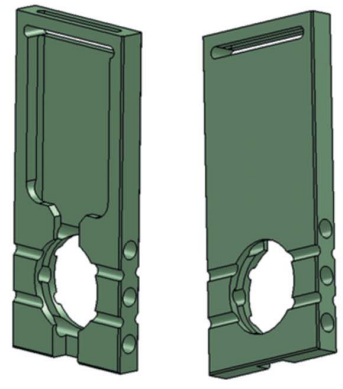

(a)

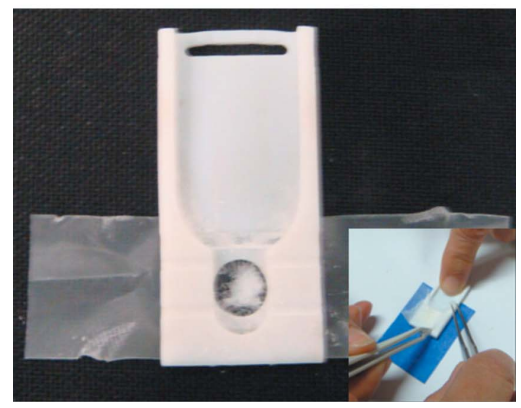

(b)

Figure 2

(a) Powder holder, front and rear views. (b) Real images of the powder mounting with the powder surrounded by the porous Teflon membrane.

for construction reasons and they are used to facilitate the cell cleaning after each experiment.

The two conical holes at the body top are used to insert a thermometer, for checking the temperature of the bath, and a reflux system, to continually cool down the vapour and return it back to the cell as a liquid. Other devices can be mounted in these holes, such as a dripping system, for example. The aperture in the middle is used to introduce the powder holder block in the liquid and it is covered with a Teflon plate, sealed by a Teflon gasket. The powder-mounting aperture is very narrow, in comparison with the body cell, reaching up to $4.5 \mathrm{~mm}$ width (see Fig. 1d). The powder holder system is fixed with two Kapton/Teflon windows, which prevent the liquid from getting out of the cell, also sealed by a gasket. The X-ray outward window is maximized to cover a maximal angular range of $\pm 45^{\circ}$. Finally, the lateral holes allow the exit of the liquid when the chemical reaction has finished. They are blocked by Teflon windows, linked with special extendable gaskets, in case the main cell is deformed owing to the temperature rising up in the interior.

Additional channels are present in the cell to carry out the reaction at a controlled and homogeneous temperature, which are not in contact with the solvent; there are four holes coupled to the cell body and sited in the upper position, in which four cartridge heaters are located to heat the cell body, and also the liquid, in a homogeneous mode. In the lateral view, two channels are available for placing two temperature probes in order to control the temperature of the reaction. The temperature of the bath reaction can be regulated between room temperature and $493 \mathrm{~K}$. As mentioned above, the heater resistances are in direct contact with the Teflon material and the bath is heated by thermal conductivity; so, although the melting point of the Teflon is about $615 \mathrm{~K}$, the recommended bath temperature should be lower than $493 \mathrm{~K}$ to avoid deformation of the main body. However, other materials such as stainless steel, with a higher melting point than Teflon, could be used to build the main body of the cell, as in the cases of studying a reaction that takes place above $493 \mathrm{~K}$.

Once the cell is mounted, the powder sample has to be fixed and located in a particular place. A special powder holder has been designed to assure that the sample is kept in a fixed position, while the experiment is running, and the liquid bathes and goes through it. This powder holder is also built of Teflon material.

The entire cell is supported on a stainless steel platform in order to avoid the possibility that the solvent sheds onto the diffractometer tower. The cell can be moved into the X-ray beam in a very precise and reproducible way, as the diffractometer tower allows translations along the $X, Y$ and $Z$ axes, besides other movements (rotation cradles). The $\mathrm{X}$-ray patterns are recorded as a function of either time or any other parameter, such as temperature, if required.

\subsection{Powder holder set-up}

In Fig. 2 a scheme of the powder holder is shown. The powder holder has a pocket shape with a large aperture for easy powder mounting reasons. The pocket aperture is used to contain the porous membrane envelope with the powder. The powder quantity is limited to a few milligrams, since the pocket is a disc of $6 \mathrm{~mm} \times 1 \mathrm{~mm}$ thickness. However, by request (in case the powder is composed of heavy elements) it can be reduced and, in this way, decrease the X-ray absorption and keep the transmission capability of the cell. In order to enhance the liquid-solid contact area, it should not be a compacted powder.

The porous membrane can be used, if necessary, to envelop the complete powder holder block. For the test measurements presented in this work, a porous Teflon membrane with a porous size of about $12.5 \mu \mathrm{m}$ (Goodfellow FP301020) has been used, which allows the passage of the liquid through the solid sample and traps the powder in the cavity. It should be noted that the porous size of the covering membrane could be changed as a function of the grain size of the experiment material. The sample holder presents three holes of diameter $2 \mathrm{~mm}$ along the main channel direction in order to improve the liquid circulation through the powder. The recommended experimental procedure to minimize the contact time between the liquid and the powder before the first data collection is to, firstly, align the cell in the X-ray beam path, secondly, place the powder holder in the cell, and, finally, fill the cell with the liquid solvent. The incoming and outcoming windows have an aperture of $5 \mathrm{~mm}$, which is larger than the minimal X-ray beam diameter of about $500 \mu \mathrm{m} \times 500 \mu \mathrm{m}$. It is very important to stress that, in case of suspicion of space/lateral inhomogeneities in the reaction process, the X-ray beam dimensions can be enlarged up to $4 \mathrm{~mm} \times 4 \mathrm{~mm}$ to guarantee a representative averaged data collection. 


\section{X-ray measurements}

Diffraction experiments have been carried out at BM25B, where the measurements were performed in transmission mode with a monochromatic X-ray beam, set to an energy of $14.89 \mathrm{keV}$ (wavelength $0.833 \AA$ ) and with a spot size of $500 \mu \mathrm{m}$ $\times 500 \mu \mathrm{m}$. The complete reaction pathways have been followed by X-ray diffraction using a bidimensional CCD camera. The reaction temperature ranges were varied from room temperature to $453 \mathrm{~K}$ and to $373 \mathrm{~K}$ for the titanium phosphates transformations and for $\mathrm{Cu}(\mathrm{ISO})_{2}$ formation, respectively.

The resulting images were integrated using the XOP2.3 program (Sánchez del Río, 2009). The CCD detector was mounted off-center to record the weak Debye-Scherrer diffraction rings, reaching up to $0.54 \AA$ of $d$-space resolution at the edge of the detector. The CCD detector produced images with $3825 \times 1912$ pixels, as $2 \times 2$ binning was used. Diffraction data were taken at $30 \mathrm{~s}$ of exposure time to obtain a better signal-to-noise ratio for weak intensity reflections. The chosen conditions $(\lambda=0.833 \AA)$ allow a maximum reachable $2 \theta$ angle of about $50^{\circ}$ when the sample-CCD-detector distance is $197 \mathrm{~mm}$. The diffraction data were obtained in transmission mode, being the geometry of the cell designed to collect the data with both detectors. The layered titanium phosphates transformations and the crystallization of the $\mathrm{Cu}(\mathrm{ISO})_{2}$ complex were studied under the same experimental conditions.

In the case of the $\mathrm{Cu}$ (ISO) $)_{2}$ complex, XAS measurements have been performed in transmission mode by using two identical gas-filled ionization chambers just before and after the studied sample, in order to ensure the best linearity in the measurement of the photon intensity. A third ionization chamber is present in order to simultaneously measure a reference sample. XAS measurements were performed at the $\mathrm{Cu} K$-edge. Data treatment was achieved using ATHENA software. Multishell EXAFS fitting analyses were performed by shell fits on $k^{2}$-weighted Fourier-transformed spectra using the Artemis software (Ravel \& Newville, 2005).

\subsection{Synthesis route of layered titanium phosphate}

Layered titanium and zirconium phosphates have received considerable attention in the last decades. This is connected with their unique properties: high thermal and chemical stability, resistance to oxidation, selectivity to certain ions and molecules, etc, which allows the use of such materials as ion exchangers and adsorbents, molecular sieves, catalysts, ion and proton conductors, and also as convenient matrices for chemical modification (Clearfield, 1996). Two main types ( $\alpha$ and $\gamma$-phases) of layered titanium phosphates are well known, containing two phosphorus atoms per atom of group IV element. In recent years some previously unknown titanium phosphates in the form of metastable phases have been synthesized (García-Granda et al., 2010; Bortun et al., 1996; Li \& Whittingham, 1993). The synthesis and investigation of such metastable materials is of great theoretical and practical interest, because it broadens the knowledge of the formation mechanism of layered compounds in general and opens new possibilities for the preparation of novel materials with regulated and valuable properties.

The evolution of $\alpha, \beta, \gamma$ and amorphous layered titanium phosphates in boiling aqueous solutions of phosphoric acid has been studied to demonstrate the feasibility of the cell. In Figs. 3(a) and 3(b) the powder diffraction patterns show the evolution of the $\gamma$-layered titanium phosphates when the solid phase is in contact with phosphoric acid solution at 5 and $10 \mathrm{M}$
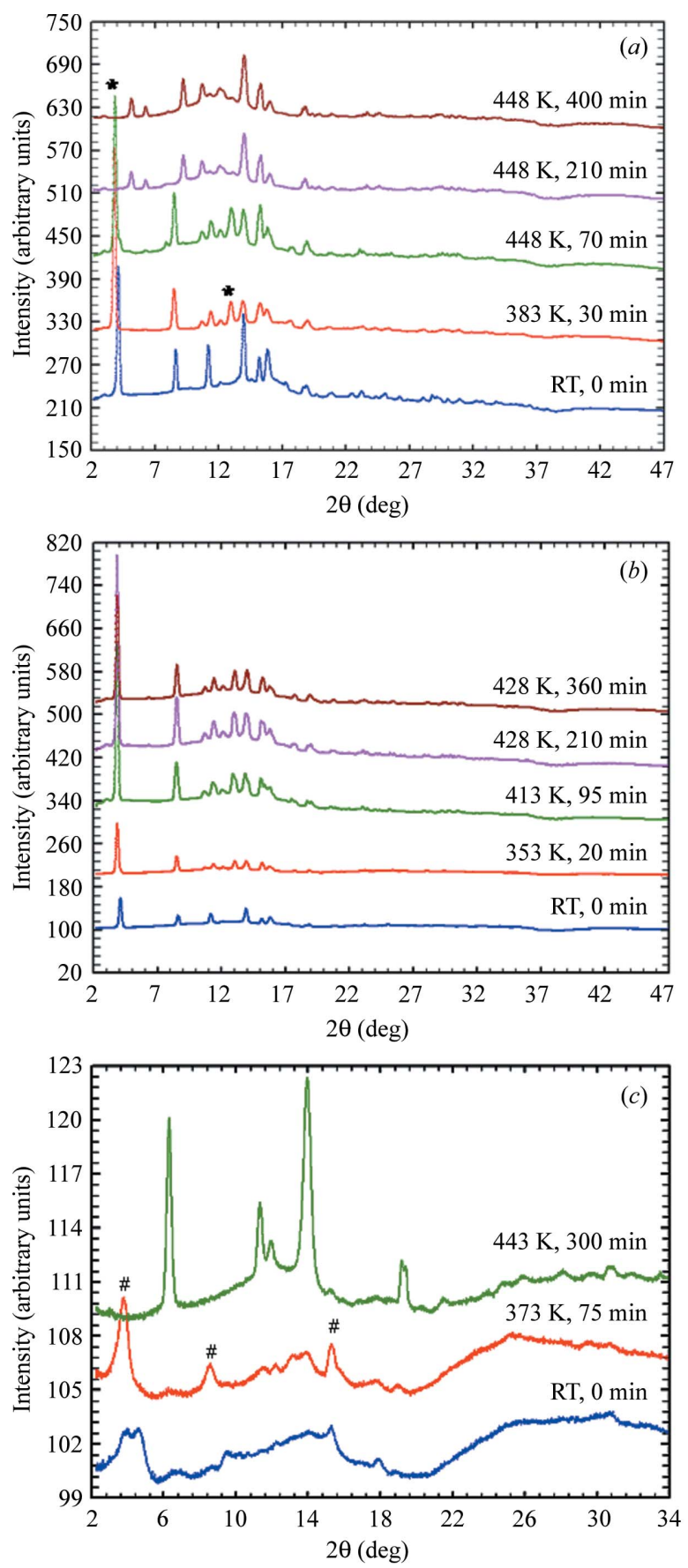

Figure 3

Reaction evolution of $\gamma$-layered titanium phosphates with (a) $5 M$ and (b) $10 M$ concentration of phosphoric acid solution. (c) Reaction evolution of amorphous titanium phosphate with $5 M$ concentration of phosphoric acid solution. (*, \#: intermediate phases; RT: room temperature.) 
concentration, respectively. The sample is placed in the sample holder and the cell is filled with phosphoric acid solution at $5 M$ concentration. The evolution of the $\gamma$-layered titanium phosphate reveals an unexpected pathway, as an intermediate phase appears after $30 \mathrm{~min}$ at $353 \mathrm{~K}$. The reaction continues until a mixture of $\beta$-Ti $\left(\mathrm{PO}_{4}\right)\left(\mathrm{H}_{2} \mathrm{PO}_{4}\right)$ and $\alpha$ - $\mathrm{Ti}\left(\mathrm{HPO}_{4}\right) 2 \cdot \mathrm{H}_{2} \mathrm{O}$ products appears. The pathway reaction of $\gamma$-layered titanium phosphate in contact with a $10 M$ concentration of phosphoric acid solution is different than the previously described one. In this synthesis route just one product is synthesized when the reaction is considered finished. Thus, the concentration degree of the phosphoric acid which is in contact with the $\gamma$-layered titanium phosphate is a critical factor in the reaction pathway. The studied reactions can be described as follows,

$$
\begin{gathered}
\gamma-\mathrm{Ti}\left(\mathrm{PO}_{4}\right)\left(\mathrm{H}_{2} \mathrm{PO}_{4}\right) \cdot 2 \mathrm{H}_{2} \mathrm{O}+5 \mathrm{MH}_{3} \mathrm{PO}_{4} \rightarrow \\
\beta-\mathrm{Ti}\left(\mathrm{PO}_{4}\right)\left(\mathrm{H}_{2} \mathrm{PO}_{4}\right)+\alpha-\mathrm{Ti}\left(\mathrm{HPO}_{4}\right)_{2} \cdot \mathrm{H}_{2} \mathrm{O}, \\
\gamma-\mathrm{Ti}\left(\mathrm{PO}_{4}\right)\left(\mathrm{H}_{2} \mathrm{PO}_{4}\right) \cdot 2 \mathrm{H}_{2} \mathrm{O}+10 M \mathrm{H}_{3} \mathrm{PO}_{4} \rightarrow \\
\gamma^{\prime}-\mathrm{Ti}\left(\mathrm{PO}_{4}\right)\left(\mathrm{H}_{2} \mathrm{PO}_{4}\right) \cdot n \mathrm{H}_{2} \mathrm{O}(n>2) .
\end{gathered}
$$

The formation of $\alpha$-titanium phosphate is also achieved starting with an amorphous titanium phosphate, as Fig. 3(c) shows. In this case the reaction has been carried out across an unknown pathway, and an intermediate phase was formed,

$$
\text { Amorphous TiP }+5 \mathrm{MH}_{3} \mathrm{PO}_{4} \rightarrow \alpha-\mathrm{Ti}\left(\mathrm{HPO}_{4}\right)_{2} \cdot \mathrm{H}_{2} \mathrm{O} \text {. }
$$

A detailed description of the synthesis route, as well as the intermediate phases, will be the subject of a forthcoming manuscript (García et al., 2012). The cell described in this work allows the study of the synthesis route of a solid in contact with a liquid. As it is shown, starting from amorphousand $\gamma$-titanium phosphate, the formation of $\alpha$-titanium phosphate can be monitored. An unknown intermediate phase has been detected; this phase is formed in the first steps of all processes and evolves in a different way as a function of the precursor materials and reaction parameters.

\subsection{Cu-isoleucine crystallization}

Copper(II) complexes of amino acids are usually considered as good model systems to attain a better insight into the characteristics of naturally occurring metalloproteins. Copper is an essential metalloelement, needed for metabolic processes in cells, and isoleucine is one of the principal structural units in metalloproteins, acting in enzymatic or structural functions. Many enzymes require copper in the active site to be biochemically active, superoxide dismutase being one of the most studied. The concentration of substrates, inhibitors, $\mathrm{pH}$ and many other factors play a crucial role on the protein's action. In order to increase the knowledge of protein's functionality, a detailed understanding of the metal amino acid bonding and bonding mechanics, for example at different $\mathrm{pH}$ values, is crucial for understanding many protein functionalities.

The crystallization reaction of copper carbonate and Lisoleucine amino acid in boiling aqueous solution has been followed by X-ray powder diffraction techniques and X-ray absorption spectroscopy. The cell performance has also been proved by following the phase transformation of copper carbonate and L-isoleucine amino acid in boiling aqueous solution. The experiment was carried out with $0.4 \mathrm{mmol}$ of $\mathrm{CuCO}_{3}$ and $0.8 \mathrm{mmol}$ of L-isoleucine (with $10 \%$ excess), which were mixed in a hand mortar and allocated in the powder holder. Once the solid mixture is allocated in the sample holder, the cell is filled with water and the reaction begins. The role of the water is to dissolve the initial reactants, and also to enhance the mobility of the ions. The chemical reaction was carried out and the formation of $\mathrm{Cu}$ (ISO) $)_{2}$ occurs. Owing to the exothermic character of the reaction and the reduced space of the sample holder, the dissolved isoleucine and $\mathrm{Cu}$ ions react, obtaining the complex formation (see Fig. 4). As the mobility of the reactants is difficult because of the presence of the porous membrane, the volume at the powder holder is oversaturated with the reaction products, producing their precipitation, that remains in the same sample holder position (i.e. the X-ray beam position) instead of flowing across the cell. This configuration allows the chemical reaction to be followed, as the initial solid and the final product remain 
in the X-ray impact position during the whole reaction process.

The diffraction study shows an immediate reaction between initial products, as the $\mathrm{Cu}(\mathrm{ISO})_{2}$ complex is observed since the first spectrum was collected, after $1 \mathrm{~min}$ in contact with water (peak at $2 \theta \simeq 4.3^{\circ}$ ). In Fig. 4 the evolution of the chemical reaction as a function of time and temperature is shown. The theoretical expected diffractogram for the reactants and product are also presented in Fig. 4 for comparison. After $1 \mathrm{~min}$, the presence of the $\mathrm{Cu}(\mathrm{ISO})_{2}$ product is observed suggesting an instantaneous chemical reaction. After $130 \mathrm{~min}$ under reflux, the initial reactant L-isoleucine is still detected on the spectra, which coincides with the excess of product added at the beginning. All of the $\mathrm{CuCO}_{3}$ reacted to achieve $\mathrm{Cu}(\mathrm{ISO})_{2}$. On the CCD images of Fig. 4 (right), the observed Debye-Scherrer rings are continuous and uniform, indicating a lack of preferential orientation, as expected.

The same reaction has been followed by XAS with the aim of showing the compatibility of the cell presented in this work. In Fig. 5 the evolution from the initial reactants to the final product is shown. Two regions show the change of the ligands around the $\mathrm{Cu}$ ion: the shape of the absorption curve presents a single peak at the beginning of the reaction, whereas a double peak can be distinguished at the end, and, a decrease on the intensity around $9060 \mathrm{eV}$. An EXAFS spectrum of the $\mathrm{Cu}(\mathrm{ISO})_{2}$ final product is shown in Fig. 6. Data are recorded after the reaction is completed, and the results are in good agreement with those expected for the $\mathrm{Cu}(\mathrm{ISO})_{2}$ complex. The separation between the different $\mathrm{Cu}$ species is, in this particular reaction, very difficult, since the oxidation state of $\mathrm{Cu}$ ions does not change appreciably between the different compounds (reactant and products). Even more, the XAS

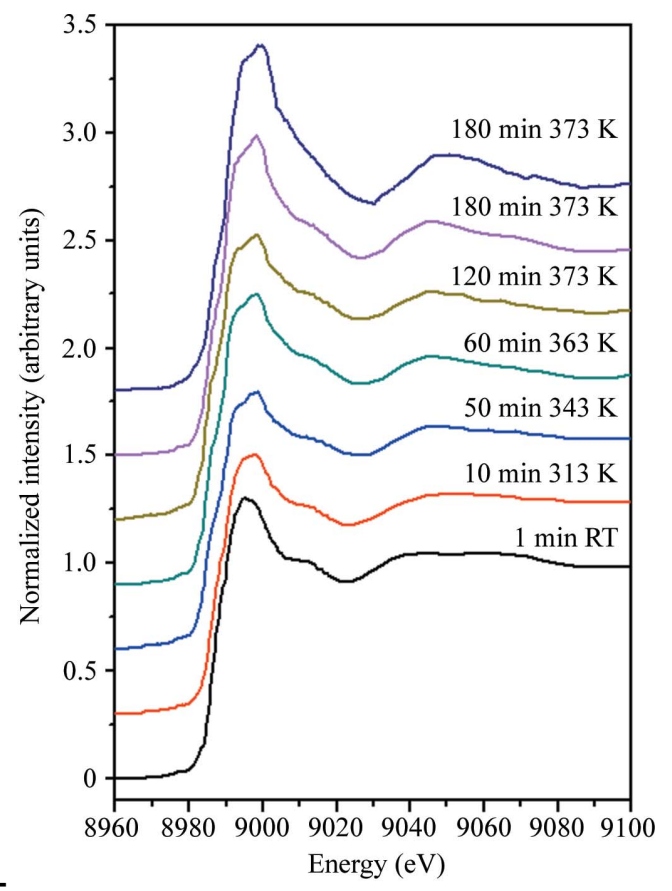

Figure 5

$\mathrm{Cu} K$-edge XANES spectra showing the evolution of the chemical reaction between $\mathrm{CuCO}_{3}$ and L-isoleucine amino acid with temperature.

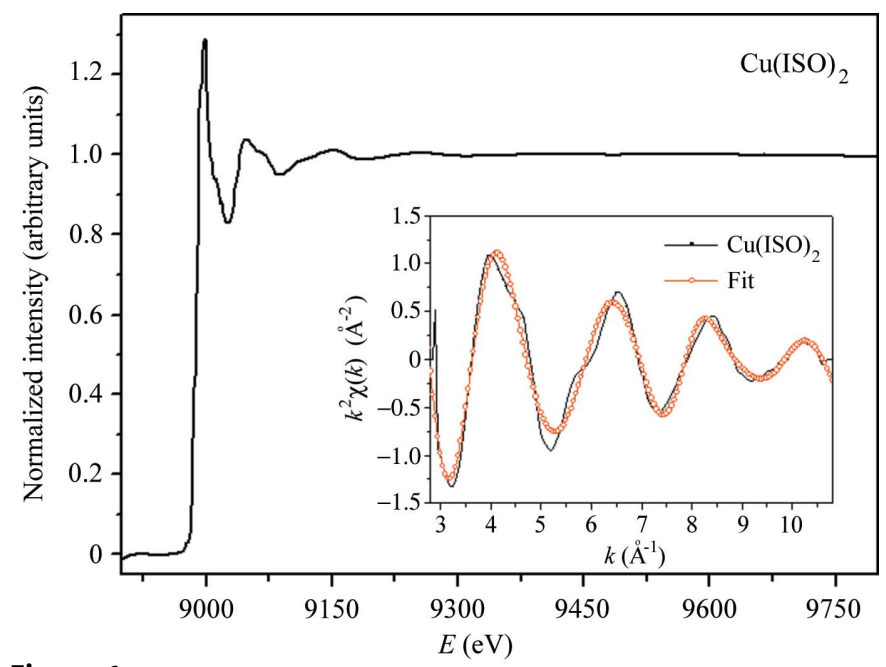

Figure 6

$\mathrm{Cu} K$-edge XAS spectra of $\mathrm{Cu}(\mathrm{ISO})_{2}$ in solid solution. Inset: EXAFS signal accounting for the first coordination shells (solid circles) and fit (open curve) for the $\mathrm{Cu}(\mathrm{ISO})_{2}$ complex.

signal is an average of present species. However, the observed changes in XANES spectra from the beginning demonstrate that the reaction takes place immediately when water comes into contact with the products.

\section{Conclusions}

A novel reaction cell has been designed to follow in situ powder-liquid reactions. Powder is mounted and placed at the $\mathrm{X}$-ray beam position inside a high-corrosion-resistant cell (Teflon material). The powder is kept in the beam position with a porous membrane, while the liquid circulates across its channels, assuring total contact with the sample. The temperature of the liquid is controlled, covering the range from room temperature up to $493 \mathrm{~K}$.

The cell feasibility has been demonstrated with two complementary experiments. The $\gamma$-layered titanium phosphate evolves to different phases depending on the concentration of phosphoric acid, which has been studied by X-ray powder diffraction. Moreover, $\mathrm{Cu}$-isoleucine complex crystallization has been studied by X-ray powder diffraction techniques and X-ray absorption spectroscopy. Crystallization processes, reaction pathways and/or phase transformations can be studied with the cell presented in this work.

We thank the SpLine staff for their assistance in using the BM25B-SpLine beamline. The financial support of the Consejo Superior de Investigaciones Científicas and Spanish Ministerio de Ciencia e Innovación (PI201060E013) is also acknowledged.

\section{References}

Alison, H. G., Davey, R. J., Garside, J., Quayle, M. J., Tiddy, G. J. T., Clarke, D. T. \& Jones, G. R. (2003). Phys. Chem. Chem. Phys. 5, 4998-5000.

Bortun, A. I., Bortun, L., Clearfield, A., Villa-García, M. A., García, J. R. \& Rodríguez, J. (1996). J. Mater. Res. 11, 2490-2498.

Castro, G. R. (1998). J. Synchrotron Rad. 5, 657-660. 


\section{research papers}

Clearfield, A. (1996). Comprehensive Supramolecular Chemistry, Vol. 7, Solid-State Supramolecular Chemistry: Two-and ThreeDimensional Inorganic Networks, edited by G. Alberti and T. Bein. New York: Pergamon.

FitzGerald, V., Drake, K. O., Jones, J. R., Smith, M. E., Honkimäki, V., Buslaps, T., Kretzschmer, M. \& Newport, R. J. (2007). J. Synchrotron Rad. 14, 492-499.

García, J. R., Alfonso, B. F., Trobajo, C., Khainakov, S. \& GarciaGranda, S. (2012). In preparation.

García-Granda, S., Khainakov, S. A., Espina, A., García, J. R., Castro, G. R., Rocha, J. \& Mafra, L. (2010). Inorg. Chem. 49, 2630-2638.

Grunwaldt, J. D., Ramin, M., Rohr, M., Michailovski, A., Patzke, G. R. \& Baiker, A. (2005). Rev. Sci. Instrum. 76, 054104.
Li, Y. J. \& Whittingham, M. S. (1993). Solid State Ion. 63, 391-395.

Norby, P. \& Hanson, J. C. (1998). Catal. Today, 39, 301-309.

Ravel, B. \& Newville, M. (2005). J. Synchrotron Rad. 12, 537-541.

Sánchez del Río, M. (2009). Synchrotron Data Analysis Using XOP, in Synchrotron Radiation in Mineralogy, Seminarios de la Sociedad Española de Mineralogía, edited by M., Suárez, E. Ayuso and E. M. Manchado, pp. 109-141. Salamanca: Sociedad Española de Mineralogía. (http://www.ehu.es/sem/revista/seminarios_m. htm\#Vol6).

Villain, F., Briois, V., Castro, I., Helary, C. \& Verdaguer, M. (1993). Anal. Chem. 65, 2545-2548.

Wall, A. J., Heaney, P. J., Mathur, R., Post, J. E., Hanson, J. C. \& Eng, P. J. (2011). J. Appl. Cryst. 44, 429-432. 